\title{
Análisis de la variabilidad geoespacial de la fragilidad morfométrica en la cuenca alta del Río Sauce Chico, Argentina
}

\section{Analysis of the geospatial variability of morphometric fragility in the upper basin of the Sauce Chico River, Argentina}

\section{Adriana C. Guzmán}

Universidad Nacional de La Plata, Argentina

\section{Lucía De Antueno}

Universidad Nacional de La Plata, Argentina

\section{Fernanda J. Gaspari *}

Universidad Nacional de La Plata, Argentina

Revista de la Facultad de Agronomía

Universidad Nacional de La Plata, Argentina

ISSN: 1669-9513

Periodicidad: Semestral

vol. 120, núm. 1, 2021

redaccion.revista@agro.unlp.edu.ar

Recepción: 07/05/2020

Aprobación: 07/06/2020

URL: http://portal.amelica.org/ameli/iatsRepo/23/232004007/index.html

DOI: https://doi.org/10.24215/16699513e069

*Autor de correspondencia: fgaspari@agro.unlp.edu.ar 


\section{Resumen}

La caracterización morfométrica permite, por medio de la definición de parámetros de forma, relieve y red de drenaje, generar una descripción de la dinámica hídrica superficial y riesgo de erosión, estableciendo su fragilidad. Esta última se expresa por los procesos degradativos que se originan de los impactos de diferente magnitud de significación espacio-temporal en cada área funcional en una cuenca hidrográfica. El objetivo del presente trabajo fue evaluar la variabilidad geoespacial de la fragilidad morfométrica de la cuenca alta del río Sauce Chico, Tornquist, Argentina. Los parámetros morfométricos se determinaron a partir de datos tomados a campo con GPS y con valores provenientes del uso de modelos de elevación digital SRTM DEM, procesados con sistemas de información geográfica. La metodología empleada permitió evidenciar que la variabilidad geoespacial de la fragilidad morfométrica varía desde las serranías hacia la desembocadura, indicando una mayor torrencialidad y con una considerable descarga de sedimentos, con una alta peligrosidad sobre los asentamientos urbanos ubicados adyacente a sus cursos de agua. Las curvas hipsométricas adimensionales indican su naturaleza sedimentaria con una fuerte expresión sobre la fase madura, en coincidencia con los factores de relieve y drenaje. Las subcuencas de la cabecera conforman el 55,33\% de cauces de orden 1 y 2 , que denota una mayor presencia de inflexiones en el relieve, por roca en superficie y menos permeable, conduciendo de forma encajonada hacia áreas de lomadas induciendo a la escorrentía en manto generada por cualquier precipitación y menor tiempo de concentración.

Palabras clave: morfometría, cuenca hidrográfica, SIG

\section{Abstract}

The morphometric characterization of a river basin allows the description of the superficial hydric dynamics and the erosion risk, by the definition of shape, relief, and drainage parameters, stablishing its fragility. This fragility is evidence at the degradative processes that originate the impacts of different spatio-temporal relevance in each functional area inside a basin. The goal of the present research was to evaluate the geo-spatial variability of the morphometric fragility in the upper basin of the Sauce Chico River, located in Tornquist, Argentina. The morphometric parameters were determined from GPS field data combined with information from digital elevation models, processed with GIS. The methodology used allowed to show the geospatial variability of morphometric fragility which varies from the headwater to the mouth, which indicate greater torrentiality and an important sediments discharge, leading to a hazardous impact in the settlements located near the water courses. The hypsometric curves indicate the sedimentary nature of this basin, with a strong expression especially in the mature phase, coinciding with for the relief and drainage parameters results. The sub-basins of the catchment area represent the $55.33 \%$ of the water channels of order 1 and 2 , this indicates a greater presence of inflections in the relief, with less permeable rock on the surface, that lead the channeled water to the hills area generating runoff as a mantle and propitiating a lower concentration time.

Keywords: morphometry, watershed, GIS 


\section{INTRODUCCIÓN}

La fragilidad ambiental se refiere al nivel de susceptibilidad de un sitio debido al efecto de algunos factores dañinos o situaciones de alto riesgo. La presencia de un sistema serrano influye en la variabilidad climática y en la dinámica hídrica en cuencas hidrográficas debido a los cambios en la morfometría de cada unidad hidrológica infiriendo fragilidad, experimentando cambios en la distribución de la cobertura vegetal. La ausencia de una planificación de uso de la cobertura vegetal del territorio intensifica los procesos de degradación ambiental en estos sitios, representados por la escasez de prácticas agrícolas con medidas conservacionistas, la recurrencia de incendios forestales, explotación turística sin planificación, que deterioran el patrimonio natural, cultural e histórico.

Las estrategias tradicionales de ordenamiento territorial (OT) suelen estar construidas de manera verticalista, con escasa y muchas veces sesgada información técnico-científica. Este tipo de estrategias suelen tener una mirada fragmentada del territorio, habitualmente focalizada en el desarrollo urbanoindustrial, basada en los intereses y necesidades de unos pocos actores, desconociendo la historia local y los beneficios y necesidades de la población (Baldini et al, 2019). Estos procesos degradativos originarán impactos de diferente magnitud intra e interzonal dando significación espacio-temporal expresando la fragilidad de cada área funcional en una cuenca hidrográfica, su transformación territorial y la dificultad de un manejo integral del agua y sus recursos naturales.

La transformación territorial está vinculada a la expansión de la agricultura sobre ecosistemas naturales, principalmente boscosos. Al pasar de coberturas naturales a usos agrarios se disminuye la cobertura del suelo, incrementando la pérdida del suelo por erosión hídrica superficial (Diaz Gómez \& Gaspari, 2018). En este contexto de vulnerabilidad ambiental, la información de precipitación también es crítica para la comprensión del equilibrio hidrológico a diferentes escalas, y para la comprensión de las interacciones complejas que existen entre los componentes del ciclo hidrológico (Gavilán et al, 2019).

En ordenamiento de cuencas hidrográficas, se plantean además, estrategias para el análisis de variables morfométricas, que expresan el nivel de fragilidad de estas unidades de gestión territorial, debido a que el conocimiento de la variación espacial de los parámetros de forma, relieve y drenaje, permite manifestar y diseñar las líneas generales del movimiento y captación del agua de lluvia (Gaspari et al., 2013; Michalijos \& Zapperi, 2015; Khurana et al., 2020); Shivanna \& Vyshnavi,2019; Venkatesh \& Anshumali, 2019).

Gran parte de los estudios realizados sobre la dinámica fluvial serrana que describen la información hidrográfica y morfológica a nivel de cuencas y subcuencas bonaerenses (Argentina), se centran en los ríos Sauce Grande, Napostá y Sauce Corto, siendo estos la principal fuente de agua dulce de la región y presentan una gran relevancia agroecosistémica. El propósito de estos estudios fue generar herramientas de gestión y ordenamiento de cuencas para disminuir los efectos de crecidas e inundaciones en poblaciones adyacentes (Hauri, 2006; Gil, 2009; Campo et al, 2016; Casado \& Campo, 2019).

Los indicadores morfométricos se determinan con el uso de técnicas de detección remota en sistema de información geográfica (SIG), mediante el análisis de modelos de elevación digital (MED - DEM por su siglas en inglés), como ser SRTM DEM (Shuttle Radar Topography Mission) ${ }^{[1]}$ generado por la National Aeronautics and Space Administration (NASA)(2000), y puntos de posicionamiento geoespacial (GPS) obtenidos en campo. Por su parte, la morfometría establece factores que pone en manifiesto las áreas que presentan fragilidad natural, que, a partir de una combinación de condiciones intrínsecas en cada cuenca hidrográfica, expresa sus problemáticas y potencialidades de carácter biofísico, como así también sus relaciones entre los múltiples usos e intereses socio productivos, base para el OT.

El objetivo del presente trabajo fue analizar la variabilidad geoespacial de la fragilidad morfométrica de la cuenca alta del río Sauce Chico, Tornquist, Argentina.

El área de estudio comprende la zona serrana en la cuenca del río Sauce Chico (CARSCH) en la región pampeana en el suroeste de la Provincia de Buenos Aires, Argentina (Figura 1). El curso principal recibe el aporte de los arroyos Sauce Chico, Barril, Ventana y San Juan, que drenan los faldeos de los cordones serranos Curamalal, Bravard y Ventana (Gaspari \& Bruno, 2003; Gaspari, 2007; Torrero, 2009; FUNS, 2011). El clima es templado a sub-húmedo seco, con una precipitación media anual de $615 \mathrm{~mm}$, con una variabilidad entre 396 a $1056 \mathrm{~mm}$. Los valores de temperatura oscilan entre 6,7 a 23,2 ${ }^{\circ} \mathrm{C}$ con una media anual de $14,5^{\circ} \mathrm{C}$ (Gaspari, 2007). Las principales actividades económicas que se desarrollan en la cuenca son ganadería y agricultura principalmente de secano, con una gran presencia de actividades turísticas recreativas. 

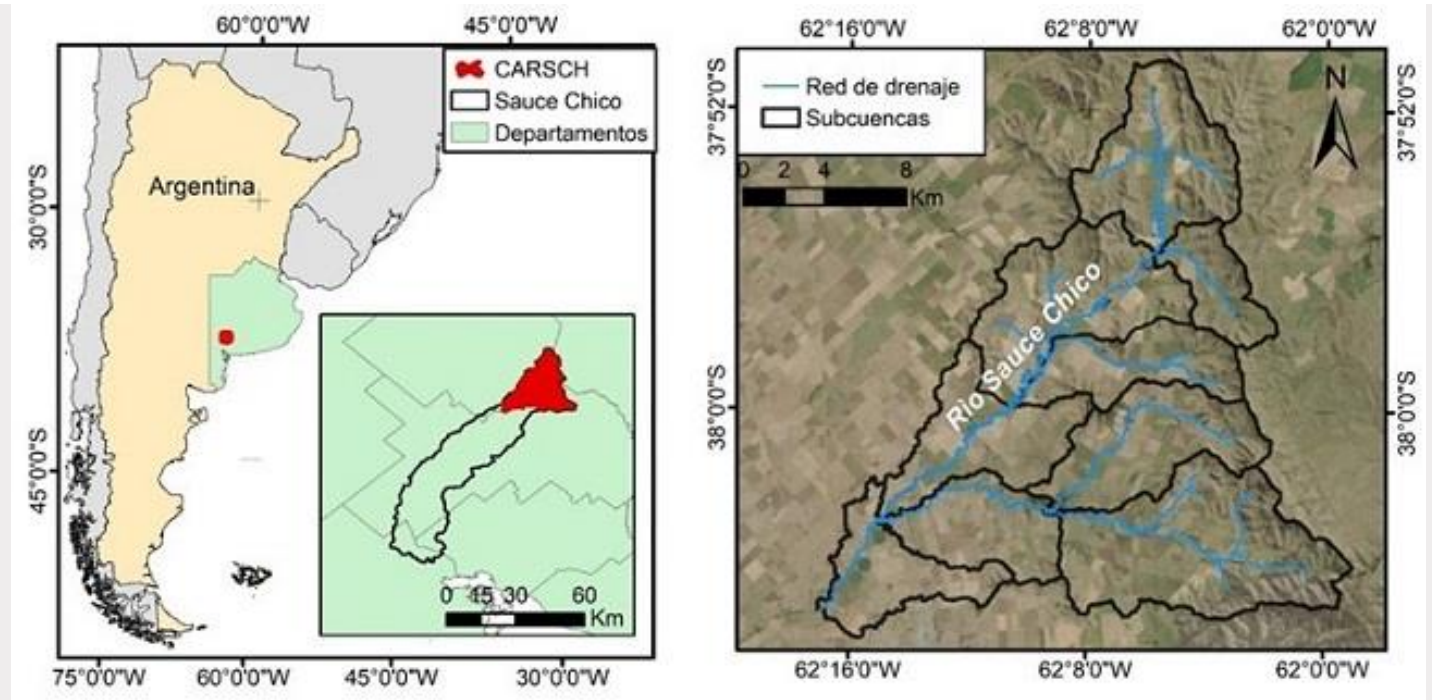

Figura 1

Ubicación del área de estudio

\section{METODOLOGIA}

La caracterización morfométrica de la CARSCH se realizó integrando metodologías aportadas por Horton (1945), López Cádenas del Llano (1998), Gaspari et al. (2013), Khurana et al. (2020) y Mandale \& Bansod (2019). La información acerca de la elevación del terreno fue obtenida de datos digitales procedentes del proyecto SRTM (2000). Este modelo digital de elevación (DEM) se empleó para la delimitación de subcuencas y la traza de seis perfiles transversales representativos del relieve, que esquematizaron la rugosidad dependiendo de la distancia entre las divisorias de agua de cada sección.

Mediante la utilización de técnicas de detección remota y sistema de información geográfica (SIG), del software QGIS@, se generó la grilla de dirección de flujo, acumulación de flujo y red hidrográfica, y se determinaron los órdenes de los cauces que conforman la red de drenaje, aplicando una rutina de cálculo en el área de estudio, los cuales fueron corroborados a campo. Se ajustaron estos cursos fluviales por medio de la digitalización de manera manual y a partir del reconocimiento y análisis del historial de imágenes satelitales de Google Earth (C) (30 de diciembre de 1984 al 19 de septiembre de 2019).

La cuantificación morfométrica se aplicó con la herramienta de GRASS del QGIS@, estableciendo los datos base para determinar los parámetros de forma, relieve (Tabla 1) y drenaje (Tabla 2).

\section{PARÁMETROS DE FORMA}

Se calcularon la superficie de ocupación de la cuenca y de cada subcuenca de la CARSCH (área)(A); el perímetro $(\mathrm{P})$ definido como la longitud de la línea envolvente de cada subcuenca sobre la divisoria de aguas; la Longitud axial (La) determinada como la distancia entre la desembocadura y el punto más lejano de la cuenca; el Ancho máximo (a) y el promedio (AP) expresado por el cociente entre el área de la cuenca y su longitud axial; el Factor de forma de la cuenca $(\mathrm{Ff})$ que comprende la distribución o arreglo geométrico de los ríos tributarios que integran la red hidrográfica; la Relación de elongación (Re) que expresa la relación entre el diámetro (D) de un círculo que tiene la misma área que cada subcuenca y la longitud máxima de la misma. También se estableció la Relación circulatoria $(\mathrm{Rc})$, que es la relación del área de una subcuenca al área de un círculo que tiene la misma circunferencia que su perímetro, el Índice de homogeneidad $(\mathrm{IH})$, que indica la relación entre longitud axial y el ancho máximo, y el de alargamiento (la) que se refiere al cociente entre el área de cada subcuenca y la relación entre longitud axial y ancho promedio. Finalmente, se cuantificó el Coeficiente de Compacidad de Gravelius (Kc) que permitió relacionar el perímetro de cada subcuenca con el de un círculo de área equivalente al de la misma, reflejando la importancia de la forma y la superficie que abarca la cuenca vertiente sobre los escurrimientos y la marcha del hidrograma resultante de una precipitación. 
Tabla 1

Parámetros morfométricos a nivel de subcuenca hidrográfica

\begin{tabular}{|c|c|c|c|c|}
\hline & Parámetro & Unidad & Ecuación & Referencia \\
\hline \multirow{12}{*}{ 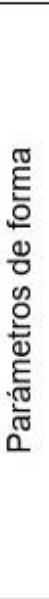 } & Superficie (A) & $\mathrm{km}^{2}$ & & Gaspari et al. (2013) \\
\hline & Perímetro $(P)$ & $\mathrm{km}$ & & Gaspari et al. (2013) \\
\hline & Longitud axial (La) & $\mathrm{km}$ & & Horton (1945) \\
\hline & Ancho máximo (a) & $\mathrm{km}$ & & Gaspari et al. (2013) \\
\hline & Ancho promedio (AP) & $\mathrm{km}$ & $\mathrm{A} / \mathrm{La}$ & Gaspari et al. (2013) \\
\hline & Factor de forma $(\mathrm{Ff})$ & & $\mathrm{A} / \mathrm{La}^{2}$ & Horton (1945) \\
\hline & Diámetro (D) & $\mathrm{km}$ & $(\mathrm{A} / \pi)^{1 / 2 * 2}$ & Strahler (1964) \\
\hline & Relación de elongación (Re) & & $\mathrm{D} / \mathrm{La}$ & Schumm (1956) \\
\hline & Relación de circularidad (Rc) & & $4 \pi \mathrm{A} / \mathrm{P}^{2}$ & Strahler (1964) \\
\hline & Índice de homogeneidad $(\mathrm{IH})$ & & $\mathrm{La} / \mathrm{a}$ & Cruz el al (2015) \\
\hline & Índice de alargamiento (la) & & $\mathrm{A} /(\mathrm{La} * \mathrm{a})$ & Horton (1945) \\
\hline & Índice de Gravelius (Kc) & & $0,284 * \mathrm{P} / \mathrm{A}^{0,5}$ & Gravelius (1914) \\
\hline \multirow{7}{*}{ 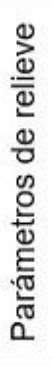 } & Elevación máxima (Hmax) & msnm & & Gaspari et al. (2013) \\
\hline & Elevación mínima (Hmin) & msnm & & Gaspari et al. (2013) \\
\hline & Desnivel $(\mathrm{H})$ & msnm & $\mathrm{H}_{\max }-\mathrm{H}_{\min }$ & $\begin{array}{c}\text { Hadley \& Schumm } \\
\text { (1961) }\end{array}$ \\
\hline & Pendiente media (PM) & $\%$ & & Gaspari et al. (2013) \\
\hline & Relieve relativo (Rr) & & $\mathrm{H} / \mathrm{P}$ & Schumm (1963) \\
\hline & Relación relieve (Rh) & & $\mathrm{H} / \mathrm{La}$ & Schumm (1956) \\
\hline & Coeficiente de rugosidad (Ra) & & $\mathrm{H} / \mathrm{Dd}$ & $\begin{array}{l}\text { López Cadenas del Llano } \\
\text { (1998) }\end{array}$ \\
\hline
\end{tabular}

\section{PARÁmetros de RELIEVE}

Se estableció la Elevación máxima (Hmax) y mínima de cada subcuenca (Hmin); el Desnivel $(\mathrm{H})$ establecido como la diferencia entre la elevación máxima y la mínima (cota); la Pendiente media de la cuenca (PM), precisada a partir del DEM con la herramienta Estadística de Zonas, con QGISC); el Relieve relativo (Rr) que es el cociente entre el máximo desnivel de cada subcuenca y su perímetro; la Relación relieve $(\mathrm{Rh})$ que se determinó como el cociente entre el máximo desnivel de cada subcuenca y su longitud axial y, por último, el Coeficiente de rugosidad $(\mathrm{Ra})$ definido como el cociente entre el relieve total $(\mathrm{H})$ y la densidad de drenaje (Dd). Otro factor de relieve importante analizado, fue la Curva hipsométrica (CH), que es una representación gráfica de la distribución del área de acuerdo con su altitud, expresada según Strahler (1957) y Langbein (1947), en una curva de doble eje de coordenadas, cuya ordenada es la cota (msnm) y la abscisa es el área por encima de una cota dada (en \% o km²). La CH se obtuvo a partir del geoprocesamiento del DEM para cada subcuenca, con SIG.

\section{PARÁmetros de LA RED de dRENAJE A NIVEL DE CUENCA HIDROGRÁFICA}

Corresponden a la distribución o arreglo geométrico de los ríos tributarios que conforman cada unidad hidrológica (Gaspari et al., 2013), representados por la Longitud total de la red de drenaje (Lu) determinada por la sumatoria de la longitud de todos los cursos de agua que conforman la red de drenaje; la Densidad de drenaje (Dd) definida como el cociente entre la suma de la longitud de todos los cursos de agua (Lu) y el área de la cuenca (A); la Longitud del cauce principal (L); la Elevación máxima y mínima del cauce (cota) 
(msnm) con las que se determinó la pendiente media del cauce principal en cada subcuenca (PMC). Para la determinación del máximo orden de cauce se empleó el método Strahler (1964), para lo cual se cuantificó la cantidad de cauces ( $\mathrm{Nu}$ ) y la frecuencia de cauces (Fs) por subcuenca siendo este un cociente entre la cantidad por unidad de superficie. La textura de drenaje (T) representa la relación entre la Dd y la Fs. Por último, se calculó el tiempo de concentración (Tc) según Kirpich (1940), que se define como el tiempo que tarda en llegar a la sección de salida la gota de lluvia caída en el extremo hidráulicamente más alejado de la cuenca. Para ello se supone que el tiempo de duración de la lluvia es por lo menos igual al tiempo de concentración y que se distribuye uniformemente en toda la cuenca. Este parámetro tiene relación directa con el gasto pico y con el tiempo de recesión de la cuenca (Tc muy cortos tienen gastos picos intensos y recesiones muy rápidas; en cambio, los tiempos de concentración más largos determinan gastos, pico más atenuados y recesiones sostenidas en el tiempo) (Gaspari, 2007).

Tabla 2

Parámetros de drenaje a nivel de cuenca y por orden de cauce

\begin{tabular}{|c|c|c|c|c|}
\hline & Parámetro & Unidad & Ecuación & Referencia \\
\hline \multirow{11}{*}{ 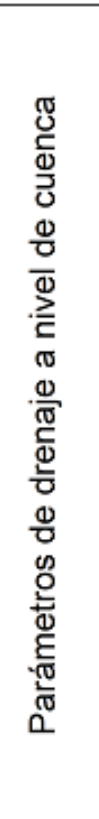 } & Longitud total red de & km & & Horton (1945) \\
\hline & $\begin{array}{l}\text { Densidad de drenaje } \\
\text { (Dd) }\end{array}$ & $\mathrm{km} / \mathrm{km}^{2}$ & $\mathrm{Lu} / \mathrm{A}$ & Horton (1945) \\
\hline & $\begin{array}{l}\text { Longitud del cauce } \\
\text { principal }(L)\end{array}$ & $\mathrm{km}$ & & $\begin{array}{c}\text { Gaspari et al. } \\
\text { (2013) }\end{array}$ \\
\hline & $\begin{array}{c}\text { Elevación máxima } \\
(\mathrm{HCmax})\end{array}$ & msnm & & $\begin{array}{c}\text { Gaspari et al. } \\
(2013)\end{array}$ \\
\hline & $\begin{array}{l}\text { Elevación mínima } \\
\qquad(\mathrm{HCmin})\end{array}$ & $\mathrm{msnm}$ & & $\begin{array}{l}\text { Gaspari et al. } \\
\text { (2013) }\end{array}$ \\
\hline & $\begin{array}{l}\text { Pendiente media del } \\
\text { cauce (PMC) }\end{array}$ & $\%$ & & $\begin{array}{c}\text { Gaspari et al. } \\
(2013)\end{array}$ \\
\hline & $\begin{array}{l}\text { Máximo orden de } \\
\text { cauce }\end{array}$ & & & Strahler (1964) \\
\hline & $\begin{array}{c}\text { Cantidad de cauces } \\
(\mathrm{Nu})\end{array}$ & & & Horton (1945) \\
\hline & $\begin{array}{l}\text { Frecuencia de cauce } \\
\qquad(\mathrm{Fs})\end{array}$ & & $\mathrm{Nu} / \mathrm{A}$ & Horton (1945) \\
\hline & Textura de drenaje $(T)$ & & Dd*Fs & Smith (1950) \\
\hline & $\begin{array}{c}\text { Tiempo de } \\
\text { concentración (Tc) }\end{array}$ & hora & $0,0078^{*}\left(\left(\mathrm{~L}^{*} 3280,8\right)^{\left.0,77 *(\mathrm{PMC} / 100)^{-0,385}\right) / 60}\right.$ & Kirpich (1940) \\
\hline \multirow{6}{*}{ 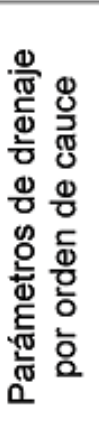 } & $\begin{array}{l}\text { Longitud de drenaje } \\
\text { (Lu) }\end{array}$ & km & & $\begin{array}{c}\text { Gaspari et al. } \\
\text { (2013) }\end{array}$ \\
\hline & $\begin{array}{c}\text { Cantidad de cauces } \\
(\mathrm{Nu})\end{array}$ & & & Horton (1945) \\
\hline & $\begin{array}{c}\text { Longitud promedio } \\
(\text { Lsm) }\end{array}$ & $\mathrm{km}$ & $\mathrm{Lu} / \mathrm{Nu}$ & Strahler (1964) \\
\hline & $\begin{array}{l}\text { Relación de longitud de } \\
\text { flujo (RL) }\end{array}$ & & $\mathrm{Lu} / \mathrm{Lu}-1$ & Horton (1945) \\
\hline & $\begin{array}{l}\text { Relación de bifurcación } \\
\qquad(\mathrm{Rb})\end{array}$ & & $\mathrm{Nu} / \mathrm{Nu}+1$ & $\begin{array}{c}\text { Schumm } \\
(1956)\end{array}$ \\
\hline & $\begin{array}{l}\text { Relación promedio de } \\
\text { bifurcación (Rbm) }\end{array}$ & & $\Sigma \mathrm{Rb}_{\mathrm{n}} / \mathrm{n} \mathrm{Rb}$ & Strahler, (1957) \\
\hline
\end{tabular}




\section{ANÁLISIS MORFOMÉTRICO A NIVEL DE ORDEN DE LA RED DE DRENAJE}

Se basó en un análisis a nivel de subcuenca de la frecuencia de cauces (Fs), la Longitud total de la red de drenaje (Lu) y la cantidad total de cauces $(\mathrm{Nu})$ de todos los órdenes por unidad de área (A). La Longitud promedio del cauce (Lsm) es la relación entre la longitud entre Lu y Nu. La Relación de longitud de flujo $(R L)$ se obtuvo por el cociente entre la longitud promedio de flujo de cualquier orden (Lu) por la longitud promedio de flujo del siguiente orden inferior (Lu-1). La Relación de bifurcación (Rb) fue determinada como la relación entre el número de segmentos de flujo de un orden dado $(\mathrm{Nu})$ y el número de segmentos del siguiente orden superior (Nu+1). Según Chopra et al. (2005) la Relación promedio de bifurcación (Rbm) (adimensional) es el promedio de las $\mathrm{Rb}$ obtenidas para todos los órdenes de cauces y expresa el nivel de control estructural debido al desarrollo geológico y litológico, por un patrón en la red de drenaje.

\section{ANÁLISIS MORFOMÉTRICO A NIVEL DE ORDEN DE LA RED DE DRENAJE}

Se basó en un análisis a nivel de subcuenca de la frecuencia de cauces (Fs), la Longitud total de la red de drenaje ( $\mathrm{Lu}$ ) y la cantidad total de cauces $(\mathrm{Nu})$ de todos los órdenes por unidad de área (A). La Longitud promedio del cauce (Lsm) es la relación entre la longitud entre Lu y Nu. La Relación de longitud de flujo $(\mathrm{RL})$ se obtuvo por el cociente entre la longitud promedio de flujo de cualquier orden (Lu) por la longitud promedio de flujo del siguiente orden inferior ( $\mathrm{Lu}-1)$. La Relación de bifurcación (Rb) fue determinada como la relación entre el número de segmentos de flujo de un orden dado $(\mathrm{Nu})$ y el número de segmentos del siguiente orden superior (Nu+1). Según Chopra et al. (2005) la Relación promedio de bifurcación (Rbm) (adimensional) es el promedio de las $\mathrm{Rb}$ obtenidas para todos los órdenes de cauces y expresa el nivel de control estructural debido al desarrollo geológico y litológico, por un patrón en la red de drenaje.

\section{RESULTADOS}

La CARSCH tiene una superficie de ocupación (A) de 365,61 km² y de acuerdo con los parámetros de forma Kc, La y Ff, se caracteriza por ser de forma alargada - triangular, representada por dos vértices en las cabeceras, generando efluentes de longitud similar, que pueden provocar crecidas significativas (Gaspari et al., 2013; Cruz et al., 2015). La red de drenaje muestra un patrón natural dendrítico de orden 5 , con orientación norte - sur sobre un eje y el otro este - oeste, cuya densidad de drenaje es moderada con un valor de $1,13 \mathrm{~km} / \mathrm{km}^{2}$, según la clasificación de Delgadillo \& Páez (2008).

$\mathrm{La}$ CARSCH presenta elevaciones comprendidas entre 254 a $1.112 \mathrm{msnm}$, con una pendiente media de 16,6\%. El procesamiento geoespacial de la información altimétrica permitió definir la divisoria de aguas superficial de unidades hidrológicas menores, subcuencas (SC), corroborando además su sistema de drenaje superficial (Figura 2).

Los parámetros morfométricos calculados según la Tabla 1 se establecieron para las 9 subcuencas que conformaron el área de estudio (Tabla 3).

\section{PARÁMETROS DE FORMA}

Las subcuencas de la cabecera de CARSCH son las de mayor ocupación de superficie, siendo las de transición y desembocadura las más pequeñas, teniendo una relación directa con $P, D$, La y AP. En relación $\mathrm{Re}, \mathrm{Rc}, \mathrm{Ff}$ y Kc las subcuencas SC1 y SC3 presentan una menor elongación y forma oval. El resto de las subcuencas son de forma rectangular - oval y elongadas. El La indica que SC1 presenta poco alargamiento; y la más extensa es la subcuenca Barril, siendo el resto de las subcuencas moderadamente alargadas. Del análisis integrado de todos los factores se establece que las subcuencas serranas son más elongadas y las que se encuentran cercanas a la desembocadura tienden a la circularidad generando un tiempo de concentración menor con mayor posibilidad que las ondas de crecida sean continuas.

\section{PARÁmetros de RELIEVE}

En la CARSCH el terreno plano alcanza solo el $6,8 \%$ de su superficie de ocupación, el 17,6\% es de pendiente suave y el $38,1 \%$ expresa lomadas, presentando estas últimas áreas la necesidad de medidas de conservación de suelo para evitar su degradación por erosión hídrica superficial. Dentro de la cuenca existen tres áreas marcadas: la cabecera, con elevaciones superiores a $800 \mathrm{msnm}$, la zona media, de 350 a $800 \mathrm{msnm}$ y la zona baja con cotas menores $350 \mathrm{msnm}$. Las pendientes fuertes (mayores al 12\%) representan el $37,44 \%$ del área serrana de la zona estudiada (Figura 3). Según la condición altimétrica, 
las subcuencas SC1, SC2, Barril, San Juan, Ventana 1 y SC3 presentan cotas superiores a los 800 msnm, encontrándose en la cabecera de la cuenca definiendo el inicio de los principales tributarios. En cuanto a $\mathrm{H}$, el mayor se observa en las subcuencas San Juan y Ventana 1 con cotas mayores a los 770 msnm; en SC1, Barril y SC3, H oscila entre 600 y 700 msnm; la SC2 alcanza los 480 metros de desnivel; mientras que para las subcuencas Ventana 2 y SC4 presentan desniveles de $330 \mathrm{msnm}$.

Según la Tabla 3 y la Figura 3, las subcuencas SC1, SC2 y Ventana 1 presentan una pendiente media muy fuerte y con terreno accidentado en las subcuencas del Barril, San Juan y SC3. Las subcuencas del Ventana 2, SC4 y SC5 presentan una pendiente media superiores al 6\%, expresada por lomadas en las dos primeras; mientras que, en la zona baja de la SC5, la pendiente media es solo del $4,34 \%$.

Del análisis de los factores de relieve se interpreta que el $24,43 \%$ de la cuenca presenta un terreno plano - suave donde la aplicación de medidas de laboreo del suelo, como son siembra directa, labranza de conservación o labranza cero sobre rastrojo, son técnicas de cultivo apropiadas para evitar la alteración del suelo mediante arado. El 38,13\% de la superficie de la CARSCH se encuentra ocupada por lomadas (entre $3-12 \%$ de pendiente), siendo esta área la que presenta una mayor fragilidad morfométrica expresando la necesidad de implementación de medidas de conservación de suelo, para evitar su degradación y pérdida de suelo superficial. Las zonas con pendientes fuertes, mayores al $12 \%$, representan el $37,44 \%$ de la zona estudiada, por lo cual expresan sectores muy frágiles que deben tender a la conservación del paisaje, promoviendo su regeneración y protección, para evitar la disminución del valor paisajístico de la CARSCH.

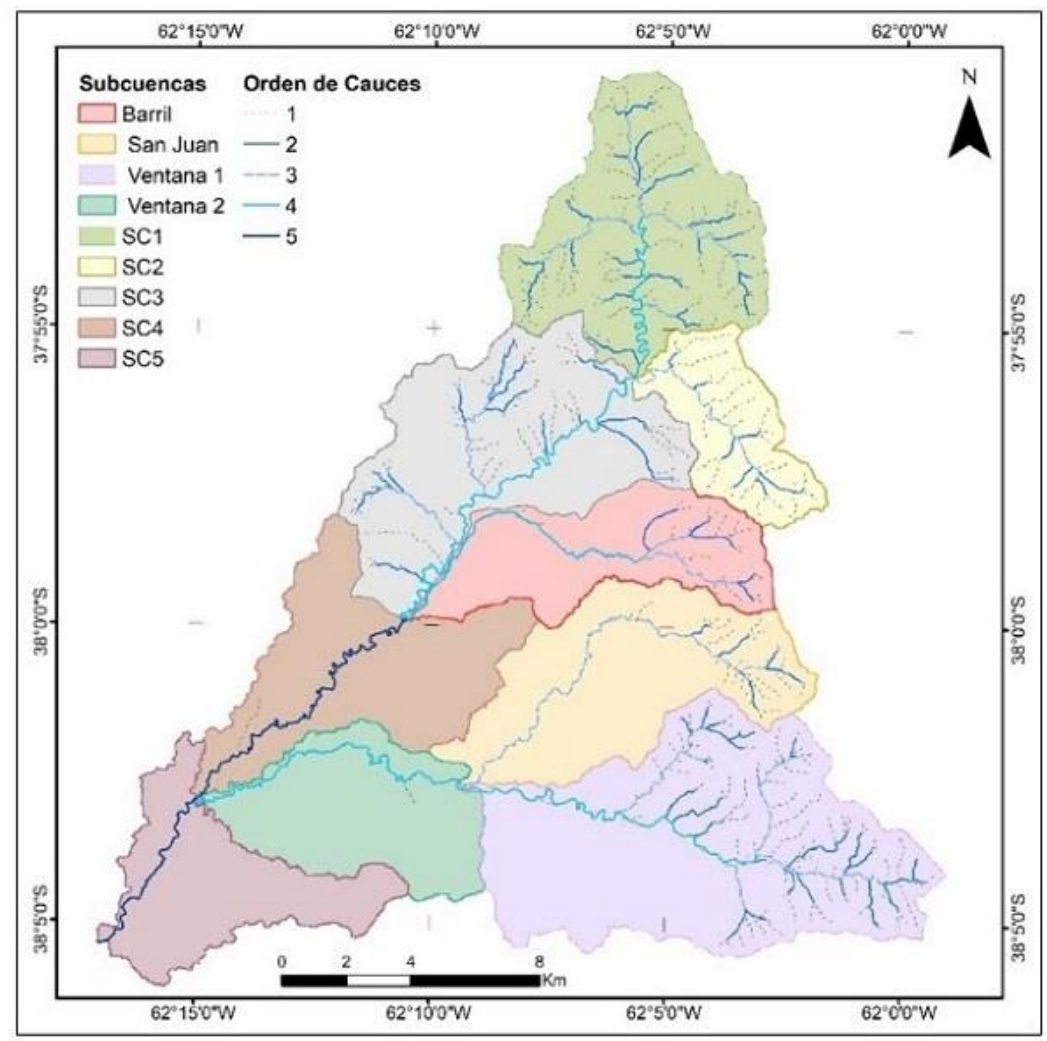

Figura 2

CARSCH: Subcuencas (SC) con su red y orden de drenaje 


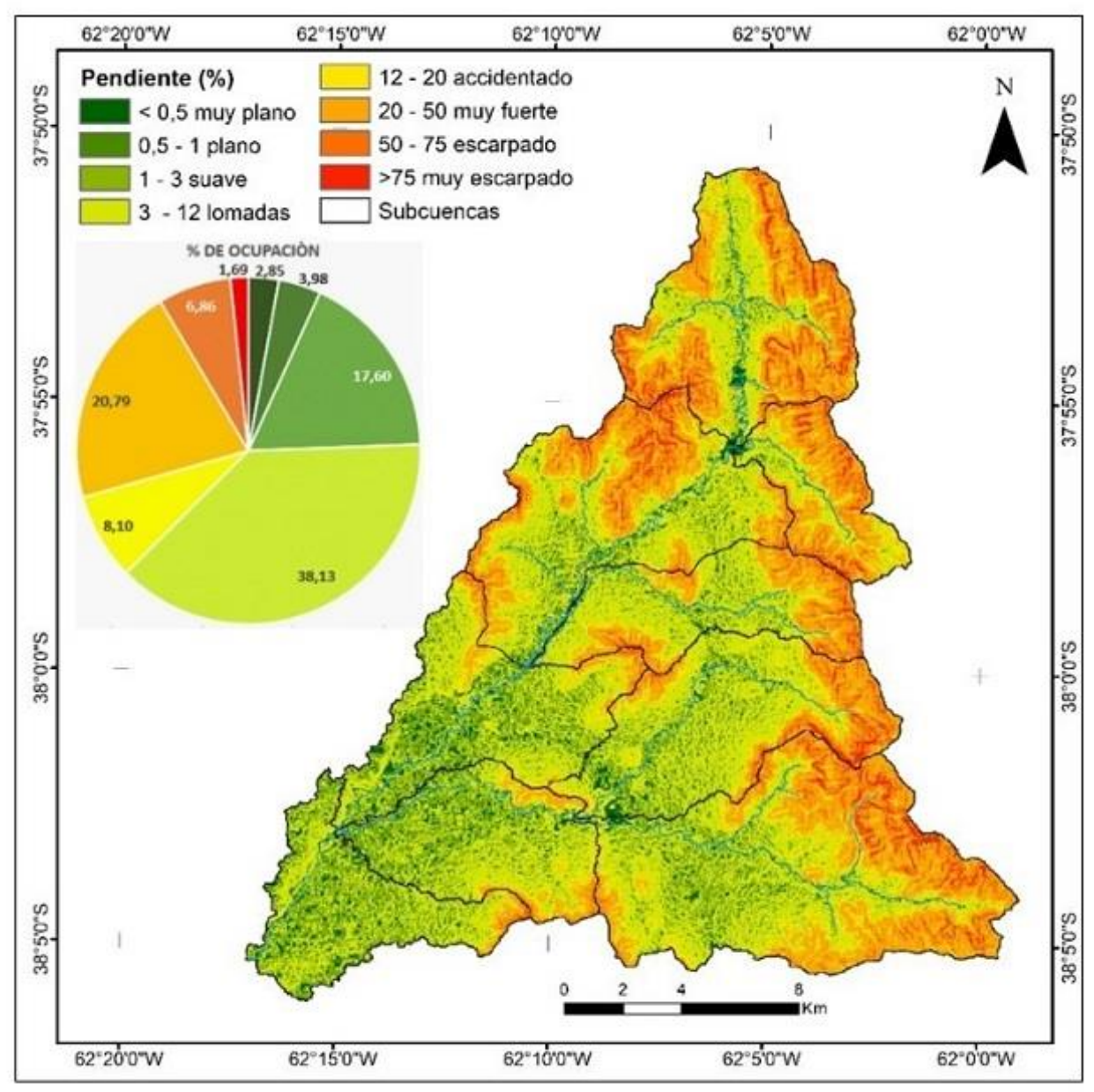

Figura 3

Mapa de pendientes y porcentaje de ocupación de la CARSCH

De acuerdo con la Tabla 3, el valor de Rr y Rh de las subcuencas SC2, San Juan y SC3 presentan mayor fragilidad morfométrica que genera una susceptibilidad de degradación por erosión hídrica superficial; las subcuencas Barril y Ventana 1 presentan riesgo moderado; finalmente SC4, SC5 y Ventana 2 tienen baja susceptibilidad. En relación con Ra este indica que la sinuosidad disminuye desde la cabecera hacia la desembocadura en cada subcuenca de la CARSCH.

La interpretación de la Figura 4, que representa la Curva hipsométrica $(\mathrm{CH})$ de cada subcuenca, permite establecer la superficie de ocupación según su cota, por ejemplo: $100 \%$ de la cuenca está por encima de los $284 \mathrm{msnm}, 50 \%$ de los $318 \mathrm{msnm}, 30 \%$ de la cuenca está entre los 318-662 msnm estableciendo el quiebre de la pendiente e inicio del área serrana; $10 \%$ de la cuenca está entre los 447 - 790 msnm, ocupando la pendiente media del área serrana, alcanzando $1091 \mathrm{msnm}$ como cota máxima.

La representación de las curvas hipsométricas adimensionales (Figura 5) muestra que las subcuencas de CARSCH son sedimentarias con una fuerte expresión sobre la fase madura. 
Tabla 3

Resultados del análisis morfométrico a de forma y relieve

\begin{tabular}{|c|c|c|c|c|c|c|c|c|c|c|c|c|}
\hline & \multirow[b]{2}{*}{ Parámetro } & \multicolumn{10}{|c|}{ Subcuenca } & \multirow{2}{*}{$\begin{array}{l}\text { Cuenca } \\
\text { Sauce } \\
\text { Chico }\end{array}$} \\
\hline & & Unidad & SC1 & SC2 & Barril & $\begin{array}{l}\text { San } \\
\text { Juan }\end{array}$ & $\begin{array}{c}\text { Ventana } \\
1\end{array}$ & SC3 & $\begin{array}{c}\text { Ventana } \\
2\end{array}$ & SC4 & SC5 & \\
\hline \multirow{12}{*}{ 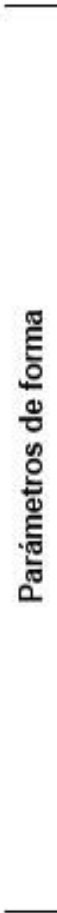 } & Superficie (A) & $\mathrm{km}^{2}$ & 46,87 & 18,89 & 30,18 & 43,59 & 75,95 & 52,57 & 30,73 & 38,53 & 28,31 & 365,61 \\
\hline & Perímetro $(\mathrm{P})$ & $\mathrm{km}$ & 42,42 & 29,00 & 37,70 & 46,95 & 60,00 & 45,92 & 37,43 & 50,00 & 48,52 & 149,79 \\
\hline & $\begin{array}{l}\text { Longitud axial } \\
\text { (La) }\end{array}$ & $\mathrm{km}$ & 9,38 & 7,29 & 11,36 & 12,30 & 15,40 & 11,33 & 9,50 & 12,06 & 9,80 & 32,35 \\
\hline & $\begin{array}{l}\text { Ancho máximo } \\
\text { (a) }\end{array}$ & $\mathrm{km}$ & 8,43 & 3,37 & 4,00 & 5,89 & 8,37 & 6,24 & 5,00 & 7,16 & 6,03 & 23,71 \\
\hline & $\begin{array}{l}\text { Ancho } \\
\text { promedio (Ap) }\end{array}$ & $\mathrm{km}$ & 5,00 & 2,59 & 2,66 & 3,54 & 4,93 & 4,64 & 3,23 & 3,19 & 2,89 & 11,30 \\
\hline & $\begin{array}{l}\text { Factor de } \\
\text { forma (Ff) }\end{array}$ & & 0,53 & 0,36 & 0,23 & 0,29 & 0,32 & 0,41 & 0,34 & 0,26 & 0,29 & 0,35 \\
\hline & Diámetro (D) & $\mathrm{km}$ & 7,73 & 4,90 & 6,20 & 7,45 & 9,83 & 8,18 & 6,26 & 7,00 & 6,00 & 21,58 \\
\hline & $\begin{array}{l}\text { Relación de } \\
\text { elongación } \\
\text { (Re) }\end{array}$ & & 0,82 & 0,67 & 0,55 & 0,61 & 0,64 & 0,72 & 0,66 & 0,58 & 0,61 & 0,67 \\
\hline & $\begin{array}{l}\text { Relación } \\
\text { circulatoria } \\
\text { (Rc) }\end{array}$ & & 0,33 & 0,28 & 0,27 & 0,25 & 0,27 & 0,31 & 0,28 & 0,19 & 0,15 & 0,20 \\
\hline & $\begin{array}{l}\text { Indice de } \\
\text { homogeneidad } \\
\text { (IH) }\end{array}$ & & 0,59 & 0,77 & 0,66 & 0,60 & 0,59 & 0,74 & 0,65 & 0,45 & 0,48 & 0,48 \\
\hline & $\begin{array}{l}\text { Indice de } \\
\text { alargamiento } \\
\text { (la) }\end{array}$ & & 1,11 & 2,16 & 2,84 & 2,09 & 1,84 & 1,82 & 1,90 & 1,68 & 1,63 & 1,36 \\
\hline & $\begin{array}{l}\text { Indice de } \\
\text { Gravelius (Kc) }\end{array}$ & & 1,75 & 1,88 & 1,94 & 2,01 & 1,94 & 1,79 & 1,90 & 2,27 & 2,57 & 2,21 \\
\hline \multirow{7}{*}{ 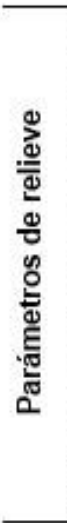 } & $\begin{array}{l}\text { Elevación } \\
\text { máxima } \\
\text { (Hmax) }\end{array}$ & $\mathrm{msnm}$ & 1.010 & 906 & 944 & 1.112 & 1.112 & 1.004 & 605 & 622 & 571 & 1.112 \\
\hline & $\begin{array}{l}\text { Elevación } \\
\text { mínima (Hmin) }\end{array}$ & msnm & 408 & 408 & 319 & 337 & 334 & 318 & 272 & 272 & 254 & 254 \\
\hline & $\begin{array}{l}\text { Relieve Total } \\
\text { (H) }\end{array}$ & $\mathrm{msnm}$ & 602 & 498 & 625 & 775 & 778 & 686 & 333 & 350 & 317 & 858 \\
\hline & $\begin{array}{l}\text { Pendiente } \\
\text { media }\end{array}$ & $\%$ & 27,16 & 29,15 & 17,35 & 14,09 & 20,56 & 19,26 & 6,52 & 6,18 & 4,34 & 16,60 \\
\hline & $\begin{array}{l}\text { Relieve } \\
\text { relativo (Rr) }\end{array}$ & & 0,01 & 0,02 & 0,02 & 0,02 & 0,01 & 0,01 & 0,01 & 0,01 & 0,01 & 0,01 \\
\hline & $\begin{array}{l}\text { Relación } \\
\text { relieve (Rh) }\end{array}$ & & 0,06 & 0,07 & 0,06 & 0,06 & 0,05 & 0,06 & 0,04 & 0,03 & 0,03 & 0,03 \\
\hline & $\begin{array}{l}\text { Coeficiente de } \\
\text { rugosidad }(\mathrm{Ra})\end{array}$ & & 0,31 & 0,27 & 0,57 & 0,94 & 0,57 & 0,47 & 0,76 & 0,86 & 1,28 & 0,76 \\
\hline
\end{tabular}




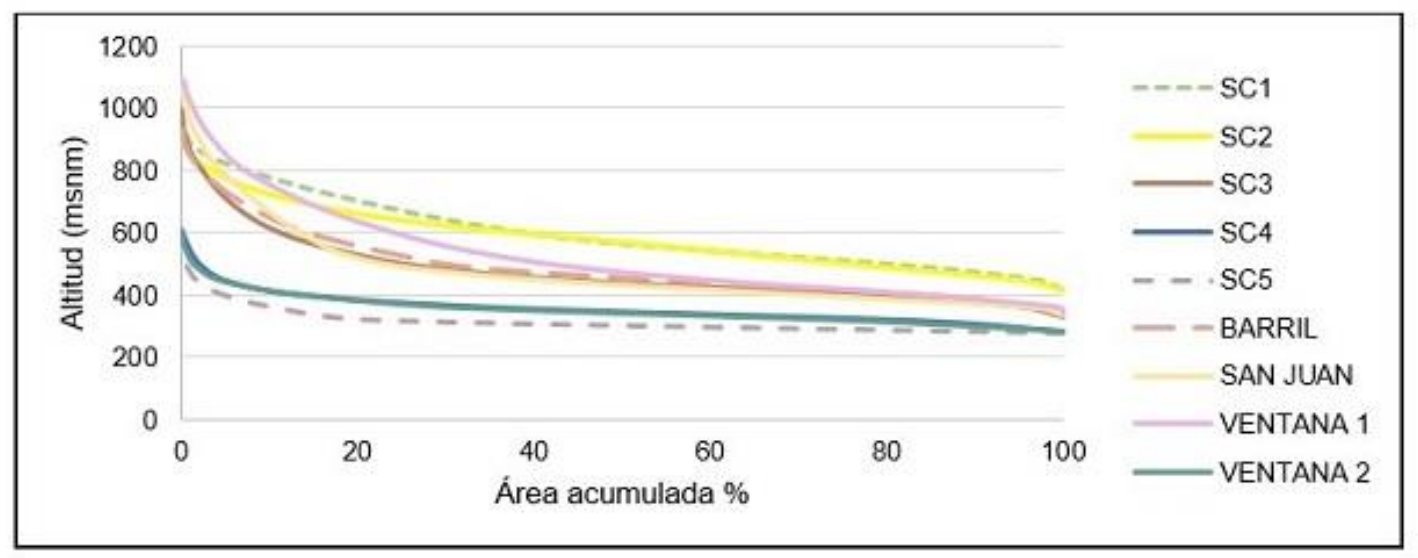

Figura 4

Curva hipsométrica por subcuenca

Los seis perfiles transversales de la CARSCH permitieron observar la correspondencia de la topografía y la fragilidad morfométrica, y al analizar la incidencia de la relación lomadas - serranía en la morfometría (Figura 6), se corroboraron los resultados alcanzados con los parámetros de relieve, que expresaron que la distribución del relieve, acompañada por el drenaje superficial, integradas a un sistema hidrológico superficial presentan fragilidad morfométrica, que disminuye desde la cabecera de cuenca hacia su desembocadura. Estos perfiles describen el número de cursos de agua permanentes y las posibles ubicaciones de drenajes transitorios ante eventos pluviales.

La representación de los perfiles transversales de la CARSCH expresa la amplitud y profundidad de los cortes erosivos fluviales e irregularidades del relieve, indicando flancos de pliegues, laderas de elevaciones o depresiones que circundan a los levantamientos de los valles, vertientes y cimas, expresando los grandes desniveles entre las divisorias de aguas y la base del terreno fluvial. Particularmente, los perfiles 1 al 4 marcan la presencia de serranías pronunciadas, y las lomadas en los perfiles 5 y 6 .

\section{PARÁMETROS DE DRENAJE}

La Tabla 4 expresa que las subcuencas SC1, Ventana 1 y SC3 presentan mayor cantidad de cauces, indicando más longitud total de la red de drenaje. Los resultados alcanzados en este estudio coinciden con las descripciones de Strahler (1964), que indica que "la alta densidad de drenaje es el resultado de material subterráneo débil o impermeable, vegetación escasa y relieve montañoso. La baja densidad de drenaje conduce a una textura de drenaje gruesa, mientras que la alta densidad de drenaje conduce a una textura de drenaje fina". Esta situación se refleja en Fs, Dd y T que expresan mayor valor en las cabeceras de cuenca disminuyendo hacia la desembocadura, indicando la relación entre la alta densidad de drenaje y la frecuencia de los cauces en las subcuencas de la cabecera de la CARSCH. El Tc de las subcuencas varía entre 1,70 y 4,56 horas, siendo el Tc de CARSCH de 8,15 horas. Las subcuencas que presentan alto Tc reflejan a una mayor longitud en el cauce principal y menor pendiente media del cauce, como por ejemplo en SC3 y SC4. Particularmente la SC2 expresa bajo Tc debido a su pendiente media moderada y corta longitud en el cauce principal. Según la Tabla 5, la diversidad de cursos de agua presentes en la CARSCH, expresan que morfométricamente cada subcuenca presenta una distribución de los órdenes de drenaje particular. Las subcuencas SC1 y Ventana 1 conforman el $55,33 \%$ de cauces de orden 1 y 2 , siendo la SC3 el $18,57 \%$ del mismo orden, que denota una mayor presencia de inflexiones en el relieve, por roca en superficie y menos permeable, conduciendo de forma encajonada hacia áreas de lomadas induciendo a la escorrentía en manto generada por cualquier precipitación, en coincidencia con Senciales (1998). La cantidad y longitud de los cauces, junto con la Dd y Fs, indican mayor grado de torrencialidad en estas subcuencas. RL indica una relación creciente de orden inferior a orden superior en todas las subcuencas, debido que se encuentran en fase madura (Figura 5). 


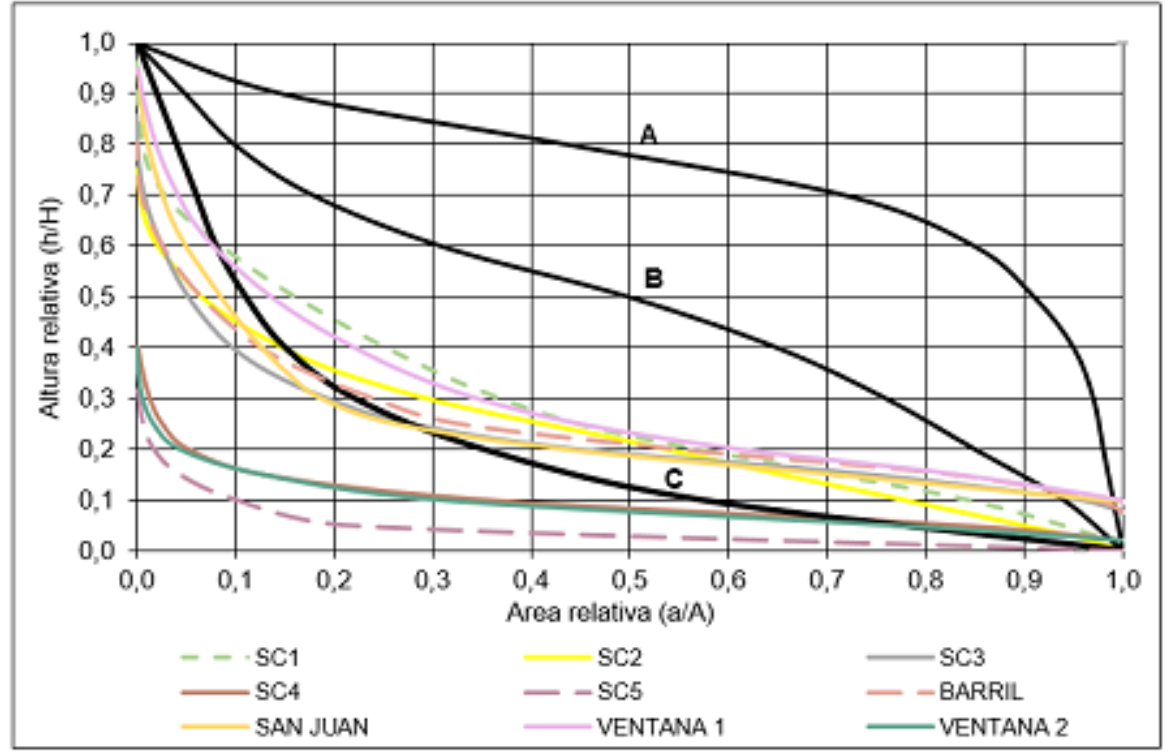

Figura 5

Curvas hipsométricas adimensionales características del ciclo de erosión en la CARSCH
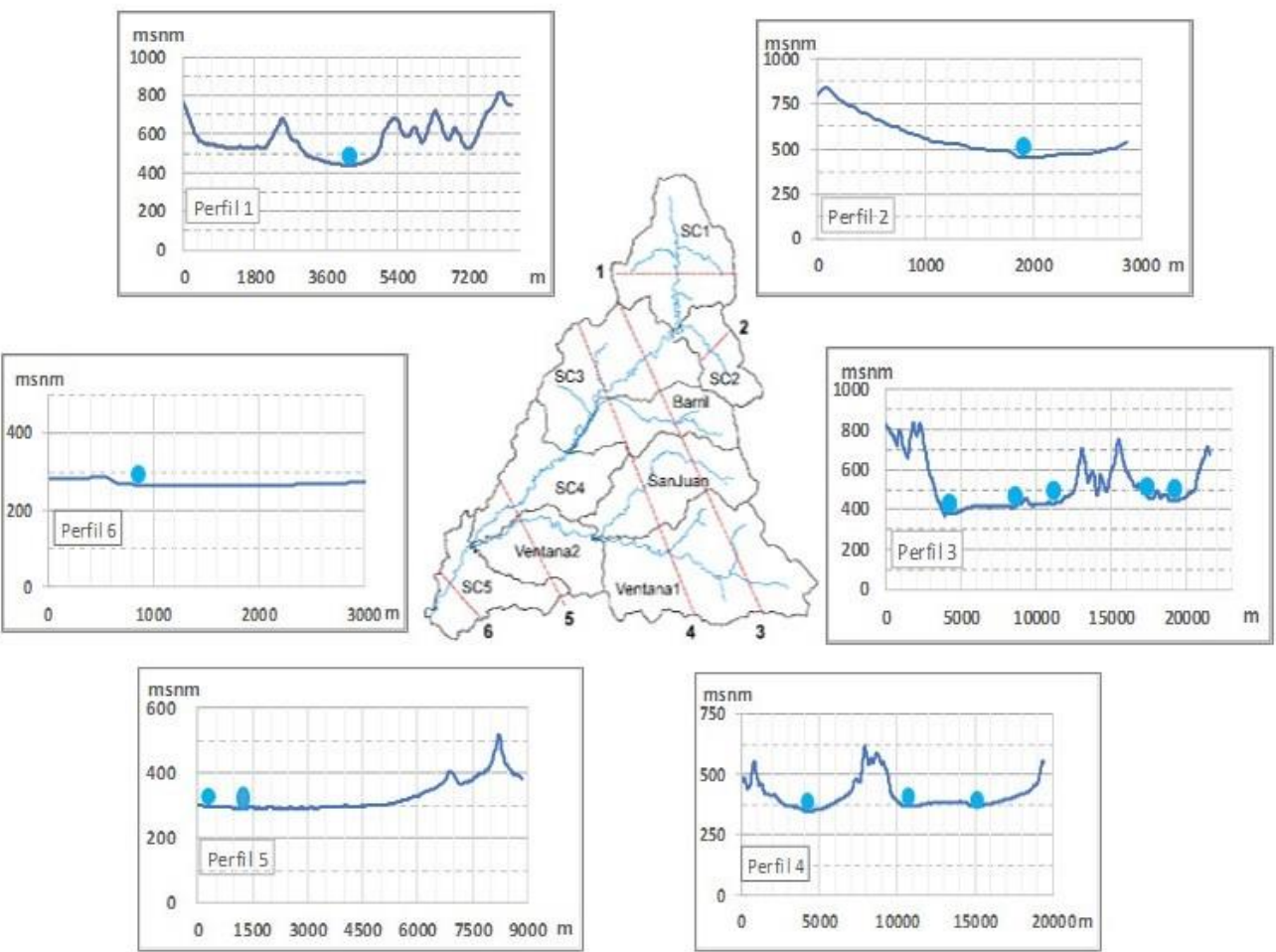

Figura 6

Perfiles transversales de la CARSCH. Los puntos celestes expresan terrenos fluviales 
Tabla 4

Análisis morfométrico de drenaje a nivel de subcuenca

\begin{tabular}{|c|c|c|c|c|c|c|c|c|c|c|}
\hline \multirow[b]{3}{*}{ Parámetro } & \multirow[b]{3}{*}{ Unidad } & \multirow{2}{*}{\multicolumn{9}{|c|}{ Subcuenca }} \\
\hline & & & & & & & & & & \\
\hline & & sc1 & $\mathrm{sc} 2$ & Barril & $\begin{array}{l}\text { San } \\
\text { Juan }\end{array}$ & $\begin{array}{c}\text { Ventana } \\
1\end{array}$ & $\mathrm{sc} 3$ & $\begin{array}{c}\text { Ventana } \\
2\end{array}$ & sc4 & sc5 \\
\hline $\begin{array}{l}\text { Longitud red } \\
\text { de drenaje } \\
\text { (Lu) }\end{array}$ & $\mathrm{km}$ & 91,23 & 34,89 & 33,17 & 35,76 & 103,63 & 76,91 & 13,40 & 15,77 & 7,02 \\
\hline $\begin{array}{l}\text { Densidad de } \\
\text { drenaje (Dd) }\end{array}$ & $\mathrm{km} / \mathrm{km}^{2}$ & 1,95 & 1,85 & 1,10 & 0,82 & 1,36 & 1,46 & 0,44 & 0,41 & 0,25 \\
\hline $\begin{array}{l}\text { Longitud del } \\
\text { cauce }\end{array}$ & $\mathrm{km}$ & 14,46 & 8.77 & 14.94 & 15,76 & 18,00 & 17,36 & 12,49 & 13,85 & 7,00 \\
\hline principal (L) & & & & & & & & & & \\
\hline $\begin{array}{l}\text { Cota máxima } \\
\text { del cauce }\end{array}$ & $\mathrm{m}$ & 800 & 551 & 540 & 512 & 508 & 414 & 335 & 321 & 278 \\
\hline $\begin{array}{l}\text { Cota mínima } \\
\text { del cauce }\end{array}$ & $\mathrm{m}$ & 408 & 408 & 319 & 337 & 334 & 318 & 272 & 272 & 254 \\
\hline $\begin{array}{l}\text { Pendiente } \\
\text { media cauce }\end{array}$ & $\%$ & $2,7 \%$ & $1,6 \%$ & $1,5 \%$ & $1,1 \%$ & $1,0 \%$ & $0,6 \%$ & $0,5 \%$ & $0,4 \%$ & $0,3 \%$ \\
\hline $\begin{array}{l}\text { Orden } \\
\text { mäximo }(\mathrm{Nu})\end{array}$ & & 4 & 2 & 4 & 4 & 4 & 5 & 4 & 1 & 5 \\
\hline $\begin{array}{l}\text { Cantidad de } \\
\text { cauces }\end{array}$ & & 156 & 70 & 51 & 52 & 168 & 126 & 4 & 2 & 1 \\
\hline $\begin{array}{l}\text { Frecuencia } \\
\text { de cauce (Fs) }\end{array}$ & & 3,33 & 3,71 & 1,69 & 1,19 & 2,21 & 2,40 & 0,13 & 0,05 & 0,04 \\
\hline $\begin{array}{l}\text { Tiempo de } \\
\text { concentración } \\
\text { (Tc) }\end{array}$ & hora & 2,93 & 1,97 & 3,27 & 3,54 & 3,64 & 3,59 & 3,43 & 3,75 & 2,39 \\
\hline $\begin{array}{l}\text { Textura de } \\
\text { drenaie }(T)\end{array}$ & & 6,48 & 6,85 & 1,86 & 0,98 & 3,02 & 3,51 & 0,06 & 0,02 & 0,01 \\
\hline
\end{tabular}

A nivel de cuenca, la relación $\mathrm{RL}$ muestra la disponibilidad de drenaje hacia el siguiente orden, estableciendo la dinámica hidrológica superficial, reflejada en el criterio de funcionar como curso transitorio (temporal), en el caso que se anula el RL. Este parámetro no se expresa en la SC5 debido a que está únicamente atravesada por el cauce principal de la CARSCH.

Las relaciones de bifurcación también se han calculado para todas las subcuencas (Tabla 5), expresando la fragilidad morfométrica de las unidades hidrológicas de la cuenca. Los valores elevados del Rbm expresan un alto nivel de control estructural (desarrollo geológico y litológico) en el patrón de la red de drenaje, mientras que los valores más bajos indican que las subcuencas están menos afectadas por las perturbaciones estructurales (Strahler, 1964; Chopra et al., 2005). Por lo cual, las subcuencas SC1, Ventana 1 y SC3 tienen un fuerte control estructural sobre el drenaje. 
Tabla 5

Análisis morfométrico de drenaje por número de orden de cauce

\begin{tabular}{|c|c|c|c|c|c|c|c|c|c|c|}
\hline \multirow[b]{2}{*}{ Parámetro } & \multirow[b]{2}{*}{ Unidad } & \multicolumn{9}{|c|}{ Subcuenca } \\
\hline & & SC1 & $\mathrm{SC} 2$ & Barril & $\begin{array}{l}\text { San } \\
\text { Juan }\end{array}$ & $\begin{array}{c}\text { Ventana } \\
1\end{array}$ & $\mathrm{SC} 3$ & $\begin{array}{c}\text { Ventana } \\
2\end{array}$ & SC4 & sc5 \\
\hline \multirow{5}{*}{$\begin{array}{l}\text { Cantidad } \\
\text { de cauces } \\
\text { (U) }\end{array}$} & 1 & 90 & 33 & 30 & 31 & 102 & 59 & 1 & 1 & \\
\hline & 2 & 51 & 15 & 14 & 17 & 58 & 42 & & & \\
\hline & 3 & 14 & 22 & 6 & 2 & 7 & 24 & & & \\
\hline & 4 & 1 & & 1 & 2 & 1 & 1 & 3 & & \\
\hline & 5 & & & & & & & & 1 & 1 \\
\hline \multirow{5}{*}{$\begin{array}{l}\text { Longitud } \\
\text { de la red } \\
\text { de drenaje } \\
\text { según } \\
\text { orden }(\mathrm{km})\end{array}$} & 1 & 43,74 & 21,58 & 10,97 & 14,58 & 48,99 & 31,75 & 0,89 & 1,92 & \\
\hline & 2 & 23,27 & 5,93 & 5,71 & 5,29 & 25,68 & 20,06 & & & \\
\hline & 3 & 15,02 & 7,10 & 5,68 & 16,05 & 15,78 & 7,74 & & & \\
\hline & 4 & 9,19 & & 10,81 & 0,07 & 13,19 & 17,36 & 12,51 & & \\
\hline & 5 & & & & & & & & 13,85 & 7,023 \\
\hline \multirow{5}{*}{$\begin{array}{l}\text { Longitud } \\
\text { promedio } \\
\text { del cauce } \\
\text { (Lsm) }\end{array}$} & 1 & 0,49 & 0,65 & 0,37 & 0,47 & 0,48 & 0,54 & 0,89 & 1,92 & \\
\hline & 2 & 0,46 & 0,40 & 0,41 & 0,31 & 0,44 & 0,48 & & & \\
\hline & 3 & 1,07 & 0,32 & 0,95 & 8,03 & 2,25 & 0,32 & & & \\
\hline & 4 & 9,19 & & 10,81 & 0,03 & 13,19 & 17,36 & 4,17 & & \\
\hline & 5 & & & & & & & & 13,85 & 7,023 \\
\hline \multirow{5}{*}{$\begin{array}{l}\text { Relación } \\
\text { de longitud } \\
\text { de flujo } \\
\text { (RL) }\end{array}$} & 1 & 1,02 & 1,05 & 1,10 & 1,07 & 1,02 & 1,03 & 0,00 & 2,08 & \\
\hline & 2 & 1,04 & 1,20 & 1,21 & 1,23 & 1,04 & 1,05 & & & \\
\hline & 3 & 1,07 & 1,16 & 1,21 & 1,07 & 1,07 & 1,15 & & & \\
\hline & 4 & 1,12 & & 1,10 & 0,00 & 1,08 & 1,06 & 1,09 & & \\
\hline & 5 & & & & & & & & 1,08 & 1,17 \\
\hline \multirow{5}{*}{$\begin{array}{l}\text { Relación } \\
\text { de } \\
\text { bifurcación } \\
(\mathrm{Rb})\end{array}$} & 1 & & & & & & & & & \\
\hline & 2 & 1,76 & 2,20 & 2,14 & 1,82 & 1,76 & 1,40 & & & \\
\hline & 3 & 3,64 & 0,68 & 2,33 & 8,50 & 8,29 & 1,75 & & & \\
\hline & 4 & 14 & & 6 & 1 & 7 & 24 & & & \\
\hline & 5 & & & & & & & & & \\
\hline $\begin{array}{l}\text { Relación } \\
\text { promedio } \\
\text { de } \\
\text { bifurcación } \\
(\mathrm{Rbm})\end{array}$ & & 6,47 & 1,44 & 3,49 & 3,77 & 5,68 & 9,05 & & & \\
\hline
\end{tabular}

\section{DISCUSIÓN}

La morfometría de una cuenca hidrográfica basa sus características en la geomorfología de base, la cual apoyada en SIG y geo-morfometría, conforman una herramienta práctica para la mitigación del riesgo a

desastres mediante la determinación de áreas susceptibles a procesos de ladera en general, como es en el caso de estudio de la CARSCH y en coincidencia con el trabajo de Román \& Castillo (2017).

Guevara Gutiérrez et al (2019) reconocen que el estudio morfométrico de cuencas fluviales, basado en jerarquización de variables que componen la red fluvial, permite sistematizar la diversidad de formas de cuencas, su tipificación y cartografía de unidades territoriales. Por ello, se establece que, en función de 
estas variables, se interpretan sus configuraciones con base en parámetros que dividen en propiedades lineales de las redes de drenaje para interpretar la función de transporte de agua, en características superficiales o área de captación pluvial que ayudan a la interpretación de la dinámica de cuencas, como sucede en el caso en estudio.

La actual deficiente planificación del territorio ignora, a su vez, el rol de las áreas naturales en la provisión de servicios ecosistémicos y en el mantenimiento de la capacidad productiva del mismo, generando problemáticas ambientales y en la reducción de estos servicios. Estas áreas naturales muchas veces consideradas como áreas "vacantes" o cuanto mucho como áreas recreativas, con bajo control de uso y/o apropiación. Como manifiesta Baldini et al (2019) la falta de una política de protección de los arroyos y ambientes riparios, ha provocado el entubamiento y/o la canalización de los arroyos cercanos al área urbana, eliminando la vegetación asociada.

Varios estudios antecedentes de Torrero (2009), FUNS (2011), Fernández et al. (2015), Espósito et al. (2016), Martínez et al. (2016), Navarro \& Cano (2017), establecen la dinámica fluvial del río Sauce Chico y sus manifestaciones de degradación y erosión hidrológicas, coincidiendo con los resultados alcanzados en el presente trabajo.

Las curvas hipsométricas adimensionales muestran que las subcuencas de CARSCH son sedimentarias con una fuerte expresión sobre la fase madura, en coincidencia con lo que indica el $R L$ y los factores de relieve.

Las características de las subcuencas de la cabecera representadas por la presencia del relieve montañoso determinan una red de drenaje más densa con mayores texturas, indicando una alta relación con la frecuencia de los cauces. Esto se evidencia en las subcuencas SC1 y Ventana 1, que conforman el $55,33 \%$ de cauces de orden 1 y 2 , siendo la SC3 el 18,57\% del mismo orden, que denota una mayor rugosidad $(\mathrm{Ra})$ y presencia de inflexiones en el relieve, por roca en superficie y menos permeable, conduciendo de forma encajonada hacia áreas de lomadas induciendo a la escorrentía en manto generada por cualquier precipitación. En dichos sectores la cantidad y longitud de los cauces, junto con la Dd y Fs, indican mayor grado de torrencialidad en estas subcuencas.

El análisis conjunto de las relaciones $\mathrm{Rr}$, $\mathrm{Rh}$ y $\mathrm{Rbm}$ expresa un alto nivel de control estructural (desarrollo geológico y litológico) en el patrón de la red de drenaje, especialmente en las subcuencas SC1, SC2, Ventana 1 y SC3 en coincidencia con un alto riesgo de degradación por erosión, mientras que en Barril y San Juan el riesgo es moderado y leve en las restantes.

La integración de todos los factores morfométricos según su distribución geoespacial expresa la fragilidad de cada subcuenca por medio de la capacidad de tolerar funciones hidrológicas potencialmente transformadoras del medio ambiente. La metodología empleada permitió evidenciar que la variabilidad geoespacial de la fragilidad morfométrica de las subcuencas estudiadas, varía desde las serranías hacia la desembocadura, siendo las SC1 y Ventana 1 las que expresan una mayor fragilidad, indicando una mayor torrencialidad y con una potencial alta descarga de sedimentos, con una alta peligrosidad sobre los asentamientos urbanos ubicados adyacentes a sus cursos de agua, por efecto de la agresividad que ejerce sobre la dinámica fluvial en esas situaciones. Por ello, cuando se expresa la fragilidad morfométrica de la CARSCH se relaciona con la sensibilidad, capacidad de adaptación y/o respuesta de la cuenca, que representa un componente frágil frente a la acción transformadora del clima y del ambiente y su distribución geoespacial.

El conocimiento de la fragilidad morfométrica de las cuencas proporciona beneficios en la relación entre sus habitantes, independientemente de que si estos, por razones administrativas, se agrupan en municipios, siendo dependientes de este sistema hídrico común, como es el caso de la CARSCH. La incorporación a la evaluación del sistema hídrico, de abordajes integrados y el apoyo de herramientas como la modelación permite identificar los cambios inducidos por acciones del hombre, sus efectos y la posible modificación de la calidad ambiental (Geraldi et al., 2010).

\section{CONCLUSIONES}

El presente trabajo constituye una descripción de la morfometría de la cuenca alta del río Sauce Chico, donde la metodología empleada, especialmente la implementación de técnicas SIG, permitió el estudio comparativo de las nueve subcuencas definidas.

Del análisis integrado de los factores de forma se establece que las subcuencas serranas, correspondientes con las ubicadas en la cabecera de la $\mathrm{CARSCH}$, son más elongadas mientras que las 
próximas a la desembocadura tienden a la circularidad, generando un tiempo de concentración menor con mayor posibilidad que las ondas de crecida sean continuas. Los parámetros de relieve y drenaje expresan que las elevadas pendientes, que dominan la cabecera y cuenca media, presentan sierras a lomadas, donde se favorece a la velocidad de los flujos superficiales, el aumento del proceso erosivo, siendo la deposición de sedimentos en la zona baja, en los valles. El patrón de drenaje está controlado principalmente por la litología y las estructuras geomorfológicas.

La detección remota y las herramientas SIG, de fácil y libre acceso, favorecieron el cálculo de los parámetros del estudio morfométrico. El aporte logrado en este trabajo conforma una base de conocimiento geoespacial de la distribución morfométrica y los estados de fragilidad en la $\mathrm{CARSCH}$, que permitirá establecer el servicio ambiental de provisión hídrica en la cuenca hidrográfica. Todos los resultados del análisis morfométrico del presente estudio se pueden utilizar para planificar el manejo de inundaciones por actividad torrencial, la evaluación de la erosión del suelo, el manejo de los recursos hídricos y el desarrollo sostenible en el área de la cuenca y plantear nuevas pautas de ordenamiento territorial, racionalizando el uso del suelo y la actividad antrópica, promoviendo el desarrollo de información geoespacial tendiendo a mejorar la productividad del territorio para el uso sustentable del agua y suelo.

\section{Agradecimientos}

Este trabajo fue financiado por el Proyecto N 11/A286 denominado "Estudio de los servicios hidrológicoambientales para aportar a la sustentabilidad de la Región Serrana Bonaerense" de la Universidad Nacional de La Plata, del Programa de Incentivos a la Investigación del Ministerio de Educación de la Nación Argentina.

\section{BIBLIOGRAFIA}

Baldini, C., M. Marasas \& A.A. Drozd. 2019. Entre la expansión urbana y la producción de alimentos. El conflicto rural/urbano en relación al patrón espacial de usos del suelo en el partido de La Plata, Buenos Aires. Revista de la Facultad de Agronomía 118(2): 1-18.

Campo, A., V. Gil, G. Ybarra \& A. Volonté. 2016. Tipificación Morfológica De Cursos Principales. Cuenca Del Arroyo El Negro, Sistema De Ventania, Argentina. Boletín Geográfico 38(1): 49-60.

Casado, A. \& A. Campo. 2019. Extremos hidroclimáticos y recursos hídricos: estado de conocimiento en el suroeste bonaerense, Argentina. Cuadernos Geográficos 58(1): 6-26.

Chopra, R., R.D. Dhiman \& P.K. Sharma. 2005. Morphometric analysis of sub-watersheds in Gurdaspur district, Punjab using remote sensing and GIS techniques. Journal of the Indian Society of Remote Sensing 33: 531.

Cruz, B., F. Gaspari, M. Vagaría, F. Carrillo \& J. Téllez. 2015. Análisis morfométrico de la cuenca hidrográfica del río Cuale, Jalisco, México. Investigación y Ciencia de La Universidad Autónoma de Aguascalientes 64: 26-34.

Delgadillo, A. \& G. Páez. 2008. Aspectos hidrológicos, subcuencas susceptibles a crecidas, escenarios de riesgo. In: Plan de desarrollo urbano del Municipio Antonio Pinto Salinas, bajo el enfoque de gestión del riesgo. Caracterización del riesgo de la cuenca del valle Mocoties. Mérida. FUNDAPRIS. Venezuela. Inédito.

Díaz Gómez, A.R. \& F. Gaspari. 2018. Transformación territorial: Intensificación agraria y pérdida del suelo en la cuenca del río Marapa, Tucumán, Argentina. Revista de la Facultad de Agronomía 116(2): 161-170.

Espósito, M., P. Zalba, D. Ribet, M. del C. Blanco \& N. Amiotti. 2016. Efectos del riego con aguas de elevados contenidos de As sobre la disponibilidad de micronutrientes. XXV Congreso Argentino de La Ciencia Del Suelo. 337 pp.

Fernández, S., M. Sequeira, M. Espósito \& J. Paoloni. 2015. Estudio del comportamiento fluviométrico e hidrológico superficial de una cuenca del sur de la Región Pampeana con datos limnigráficos diarios instantáneos: caracterización hídrica para los fines del riego.. Cuadernos del CURIHAM 21: 41-54. 
FUNS. 2011. Prefactibilidad de sistematización y aprovechamiento de la cuenca del rio Sauce Chico. $287 \mathrm{pp}$.

Gaspari, F. 2007. Plan de ordenamiento territorial en cuencas serranas degradadas utilizando sistemas de información geográfica (S.I.G.) Tesis de Maestría. Universidad Internacional de Andalucía. España. $147 \quad \mathrm{pp}$ Retrieved from https://dspace.unia.es/bitstream/handle/10334/59/0031_Gaspari.pdf?sequence=1\&isAllowed=y. Último acceso: julio de 2020.

Gaspari, F. \& J. Bruno. 2003. Diagnóstico de degradación ambiental por erosión hídrica en la cuenca del arroyo Napostá Grande. Ecología Austral 13(1): 109-120.

Gaspari, F., A. Rodríguez Vagaría, G. Senisterra, I. Delgado \& S. Besteiro. 2013. Elementos metodológicos para el manejo de cuencas hidrográficas (Primera ed). Editorial de la UNLP. 188 pp.

Gavilán, S., J.I. Pastore, J. Urang, A. Ferral, A. Lighezzolo \& P. Aceñolaza. 2019. Metodología operativa para la obtención de datos históricos de precipitación a partir de la misión satelital Tropical Rainfall Measuring Mission. Validación de resultados con datos de pluviómetros. Revista de la Facultad de Agronomía 118(1): 111-121.

Geraldi, A.M., M.C. Piccolo, \& G.M.E. Perillo. 2010. Delimitación y estudio de cuencas hidrográficas con modelos hidrológicos. Investigaciones Geográficas 52: 215-225.

Gil, V. 2009. Hidrogeomorfología de la cuenca alta del río Sauce Grande aplicada al peligro de crecidas.Tesis doctoral. Universidad Nacional del Sur. UNS. http://repositoriodigital.uns.edu.ar/bitstream/123456789/2069/1/TESIS_GIL.pdf. Último acceso: abril de 2020.

Gravelius, H. 1914. Grundrifi der gesamten Gewcisserkunde. Band 1: Flußkunde. Compendium of Hydrology 1: 265-278.

Guevara Gutiérrez, R.D., J.L. Olguín López, O.R. Mancilla Villa \& O.A. Barreto García. 2019. Análisis morfométrico de la cuenca hidrográfica del río Ayuquila, Jalisco-México. GeoFocus 24: 141139.

Hadley, R. \& S. Schumm. 1961. Sediment Sources and Drainage Basin Characteristics in Upper Cheyenne River Basin. US Geological Survey Water-Supply, 1531-B.

Hauri, B. 2006. Determinación de la erosión hídrica superficial asociada al uso del suelo en la cuenca hidrográfica del arroyo Belisario, Tornquist, Buenos Aires. FCAyF - UNLP. https://aulavirtual.agro.unlp.edu.ar/mod/page/view.php?id=979. Último acceso: julio de 2020.

Horton, R.E. 1945. Erosional development of streams and their drainage basins: Hydrophysical approach to quantitative morphology. Bulletin of the Geological Society of America 56(4): 275-370.

Khurana, D., G. Rawat, R. Raina \& R. Sharma. 2020. GIS-Based Morphometric Analysis and Prioritization of Upper Ravi Catchment, Himachal Pradesh, India. In: Advances in Water Resources Engineering and Management. Lecture Notes in Civil Engineering, vol 39, AlKhaddar R., Singh R., Dutta S., Kumari M. Ed. Springer, Singapore. pp. 163-185.

Kirpich, Z. 1940. Time of concentration of small agricultural watersheds. Civil Engineering 6: 362

Langbein, W. 1947. Topographic characteristics of drainage basins. U. S. Government Printing Office Ed. Washington, D.C.: The Superintendent of Documents.

López Cadenas de Llano F. 1998. Restauración Hidrológica Forestal de cuencas y Control de la Erosión. Ingeniería Medioambiental, TRAGSATEC, Ministerio de Medio Ambiente. Editorial Mundi Prensa. España. 945 pp.

Mandale, V. \& R. Bansod. 2019. Quantitative Morphometric Analysis of the Adula Watershed, in Ahmednagar Maharashtra Using the ESRI- ArcGIS Tool. "Advances in Water Resources Engineering and Management". Current Journal of Applied Science and Technology 36(5): 1-10.

Martínez, B.G., V. Gil, M.E. Carbone \& C.P. Simeón. 2016. Caracterización Del patrón hidromorfológico del arroyo de la ventana en su tramo medio alto (buenos aires Argentina). Estudios Geográficos 77(281): 521-541.

Michalijos, M.L. \& P. Zapperi. 2015. Análisis morfométrico aplicado a la caracterización de redes fluviales para la gestión de peligro de incendios forestales. Revista Del Departamento de Geografía 15: 53-70.

Navarro, E.L. \& D.M. Cano. 2017. Análisis morfométrico comparativo de los tramos superioresde las cuencas de los arroyos Sauce Chico, Napostá y Sauce Grande, Sierras Australes, uso de técnicas digitales. Retrieved from IV Congreso Internacional Científico y Tecnológico. CONCYT. https://digital.cic.gba.gob.ar/handle/11746/6712. Último acceso: julio de 2020. 
Román, A.Q. \& G.B. Castillo. 2017. Modelo morfométrico para determinar áreas susceptibles a procesos de ladera. Investigaciones Geográficas 94: 37-48.

Schumm, S. 1956. Evolution of Drainage Systems and Slopes in Badlands at Perth Amboy. Bulletin Geological Society of America 67: 597-646.

Schumm, S. 1963. A tentative classification of alluvial river channels. Geological Survey Circular. Ed. United States Department of the Interior (USGS). 477 pp.

Senciales, J. 1998. El análisis morfológico de las cuencas fluviales aplicado al estudio hidrográfico. Norba: Revista de Geografía 10: 155-184.

Shivanna, S. \& D.V Vyshnavi. 2019. Geomorphological study of Yelahanka watershed, Bangalore rural district, Karntaka, India. International Journal of Advanced Scientific Research and Managment 4(8): 73-77.

Smith, K. 1950. Standards for grading texture of erosional topography. American Journal of Science 248: 655-668.

Strahler, A.N. 1957. Quantitative Analysis of Watershed Geomorphology. Transactions of the American Geophysical Union 38(6): 913-920.

Strahler, A.N. 1964. Quantitative Geomorphology of Drainage Basins and Channel Networks. In: Handbook of Applied Hydrology. Chow, V. Ed. McGraw Hill. New York. pp 439-476.

Torrero, M.P. 2009. Río Sauce Chico: estudio hidrográfico para un desarrollo sustentable. Tesis doctoral. Universidad Nacional del Sur (UNS), Bahía Blanca, Argentina. 238 pp.

Venkatesh, M. \& Anshumali. 2019. A GIS-based assessment of recent changes in drainage and morphometry of Betwa River basin and sub-basins, Central India. Applied Water Science 9: 157. 